\title{
Is the salami sliced too thinly?
}

Every working scientist agrees that there is too much to read, yet most of those who make the complaint are collectively also those responsible for the common difficulty — that the literature of research is overblown.

THE origins of the simile of the scientific literature as a collection of salami slices is anybody's guess, but it is compelling. The image is a representation of the whole body of discovery as a long thin sausage, and of the items of discovery that see their way into print as the slices into which the sausage may be sliced with a sufficiently sharp knife. Of course, there are several philosophical objections; the path of discovery is not linear, for example, while many items of discovery lead nowhere, so that the corresponding salami slices may be considered to have been added in eccentric ways. But the simile has the virtue of suggesting that the process of discovery is a continuous process. Phrases such as "If I have seen further than other men, it is because I have stood on the shoulders of giants" or "One thing leads to another" apply.

The imagery is also capable of misinterpretation. For one thing, the general shape and size of the sausage can be recognized only retrospectively, after all the pieces have been added. But the most serious difficulty is that a slice of salami, like a piece of string, can be of arbitrary size. Much depends on the cutting edge of the knife and the intentions of the one who wields it. That is why the process of publication itself has been likened to that of slicing a salami. And there is a general suspicion that, as things are, the slices are too thin.

Can that be true? And how, for that matter, is it possible, at the leading edge of a growing sausage, to tell what is the appropriate thickness of a slice? Naturally, there can be no simple rule. Defining what physicists would call a lower bound could, in any case, quickly be falsified. The literature is liberally sprinkled with contributions that amount to no more than records of isolated observations - a Voyager photograph of the surface of Jupiter's moon Io, for example, or the first few records of living things at the bottoms of the deep oceans. The first account of the discovery of a pulsating star (at that stage innocent of an explanation) was similarly an intrinsically thin slice. If there should be, say, a single well-authenticated case of water running uphill, that would be a scientific observation well worthy of publication.

That is why the estimation of what constitutes a proper slice must necessarily be subjective. But even then, there are snags. It may, for example, be held that, to qualify for publication, an intended contribution to the scientific literature should be meaningful, which begs an obvious question: meaningful to whom? To close colleagues and/or competitors? To others in related fields who may profit from or be dependent on the results? Or to science more generally?

In principle, that string of questions (which may be elaborated in obvious ways) should at least be the starting point for a classification of what gets published in journals of different kinds. So far as general journals like this are concerned, for example, there is no point in the publication of material likely to interest only colleagues and competitors. (In passing, it is crucial that this does not constitute a bar to the publication of technical developments in journals such as Nature, especially as now when the adaptation of techniques developed in one field to use in another is so fruitful.) In an ideal world, what is published as original research in a journal such as this should be of more general interest than to practitioners in the single field concerned, but even that criterion does not help to define the proper salami slice.

Completeness does. These days, when the vigilance of authors on behalf of their publications seems to be matched by the vigilance of the referees for the quality of what is published, the chances that unsubstantiated observations will find their way into print diminish with the passage of time. If referees err consistently, they do so in their demands for extra information, often requiring that further experiments should be carried out. (People's failure to comply is a principal cause of casualty among the manuscripts submitted for publication.) But referees are less vigilant about completeness, and why should they be otherwise? At the best of times, they do a thankless job for nothing; why should they assess not just what an author has done, but what he might have done?

None of this implies that even the criterion of completeness is exact, although it is more satisfactory than the question about the length of a piece of string. It is almost a literary criterion. Just as it is a poor essay that does not end by answering the arresting question with which it begins, so it is much less than satisfactory that a research article should begin by listing a long string of important questions and then finish by answering only the least important of them. In that sense, most contributions to the scientific literature embody their own definitions of completeness - which should be taken more literally.

If it were indeed possible to apply this criterion rigorously, there seems very little doubt that the scientific literature would be more compact and more manageable. Sadly, experience shows that it is not so easy. For some purposes, for example, it may be sufficient to clone a gene, but for others nothing less than the nucleotide sequence will suffice. Who is to say that the first step towards the second is intrinsically unremarkable? And what is to be said of a careful demonstration that the chances that Cygnus- $\mathrm{X} 3$ is a source of gamma rays have been reduced by a further order of magnitude?

The suspicion that salami-slicing is rife has other origins, chiefly the common knowledge of the incentives that people have for behaving in this way. So long as there is a sense in which the volume of publications counts for important things - the award of research grants, promotion (for tenured people) or job security (for those on short-term contracts), the temptations must be great. Not even the bad habit of relying on citation indices will solve the problem. Indeed, it would be an interesting exercise to discover whether, when people have published two essentially similar but not identical research articles, they have succeeded somehow in doubling their citation record.

The conventional remedy for this state of affairs is that journals should be more vigilant, but there are serious limits to what journals can accomplish on their own. The ideal would be that academic institutions should find more subtle and just ways of assessing the merits of those who belong to them except by some measurement of the volume of publications, but the chances of that coming about are small. Perhaps the most urgent need is to find some means of subverting the cruel rule that, in discovery, no purpose is served in being second with even a tiny gobbet of discovery. Quite apart from its injustice, that is the greatest single incentive to premature publication. But journals which try to persuade authors of similar discoveries to lump their research reports together would not get very far.

John Maddox 